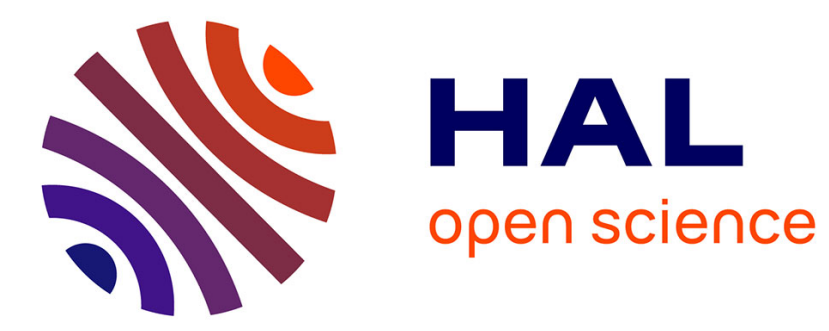

\title{
Les commerciaux et les réseaux sociaux: vers de nouveaux outils de management des ventes
}

Pascal Brassier

\section{To cite this version:}

Pascal Brassier. Les commerciaux et les réseaux sociaux: vers de nouveaux outils de management des ventes. Revue management \& avenir, 2008, 16, pp.140-154. hal-02382943

\section{HAL Id: hal-02382943 \\ https://hal.uca.fr/hal-02382943}

Submitted on 27 Nov 2019

HAL is a multi-disciplinary open access archive for the deposit and dissemination of scientific research documents, whether they are published or not. The documents may come from teaching and research institutions in France or abroad, or from public or private research centers.
L'archive ouverte pluridisciplinaire HAL, est destinée au dépôt et à la diffusion de documents scientifiques de niveau recherche, publiés ou non, émanant des établissements d'enseignement et de recherche français ou étrangers, des laboratoires publics ou privés. 
Les commerciaux et les réseaux sociaux : vers de nouveaux outils de management des ventes.

\section{Par Pascal BRASSIER}

Enseignant-chercheur au Groupe ESC Clermont, Centre de Recherche Clermontois en Gestion et Management (CRCGM), pascal.brassier@esc-clermont.fr 


\section{Les commerciaux et les réseaux sociaux : vers de nouveaux outils de management des ventes.}

\section{Par Pascal BRASSIER ${ }^{1}$}

Résumé Les acteurs commerciaux personnifient à la fois une frontière et un pont entre leur entreprise et le marché. Pourtant, les liens qu'ils tissent pour assumer ce rôle ne font l'objet que de rares recherches, y compris en gestion. Cette voie du réseau social peut, d'une part, éclairer les évolutions de la vente moderne d'un regard renouvelé. Elle peut, d'autre part, fournir des instruments explicatifs de la performance commerciale.

Le marketing relationnel a ouvert la porte d'une approche de la vente par l'angle de la relation, sans approfondir ce regard au plan interindividuel. Or, une analyse du comportement social des vendeurs suggère que la relation interpersonnelle est un facteur influent sur ses résultats.

Dans cet article, qui se veut un propos d'étape, nous précisons tout d'abord le contexte d'activité et le type de commerciaux dont nous parlons. Puis nous faisons un point sur les notions de capital social et de réseaux sociaux. Cela nous conduit à voir les réseaux sociaux comme un outil intuitivement employé depuis longtemps par ces professionnels, et à poser un certain nombre de questions à visée opérationnelle, sur la manière dont le commercial construit, développe et exploite son «portefeuille relationnel » au mieux de ses intérêts, et des intérêts de ses partenaires.

Abstract People working in sales forces represent in the same time a boundary and a bridge between their firm and the marketplace. However, the links they weave to take on their role are very rarely studied, even in management. However, on one hand, social networks offer a new way to enlighten the evolutions of selling activities. On the other hand, it may provide new instruments to explain sales performance.

Relational marketing helps to understand that relationships are a key factor for sales, but it doesn't develop the inter-individual level. An analysis of salesperson's social behaviour suggests that interpersonal relationships have an influence on sales results.

In this article, which shows a work in progress in a larger research project, we precise first the professional context and the kind of salespeople we are talking about. Next, we give a synthesis of the notions of social capital and social networks. It leads us to see social networking as an intuitive tool used for a long time by salespersons. Finally, several questions appear, with a concrete ambition: how do a salesrep act to build, develop and use his "relational portfolio" in order to optimize his interests, and his partners' ones?

\footnotetext{
${ }^{1}$ L'auteur tient à remercier son collègue Marc Lecoutre pour sa contribution de grande valeur à ce projet de
} recherche, en partie conjoint. 


\section{Introduction.}

L'acteur commercial est un acteur au rôle-frontière, dont une particularité est l'implication à la fois dans et hors de son organisation. Pour autant, sa capacité à générer et à exploiter les liens qu'il tisse avec ses nombreux interlocuteurs est un sujet peu prisé des chercheurs, en sciences de gestion notamment. Il y a pourtant là matière à apporter un éclairage nouveau sur, d'une part, la façon dont la vente et la négociation ont évolué en profondeur au cours des dernières années, et, d'autre part, l'influence que cela peut avoir sur les performances des négociateurs commerciaux.

Le cadre du marketing relationnel nous permet de situer l'importance d'une approche de la négociation commerciale par les réseaux sociaux. L'approche relationnelle est un aspect négligé de l'analyse de la vente, que Jönsson qualifie de processus social dynamique (2005), alors qu'une analyse du comportement social des négociateurs commerciaux montre qu'il s'agit effectivement d'un outil influençant ses résultats.

Nous précisons tout d'abord de quel type de vendeur, ou négociateur commercial, nous parlons, ainsi que son contexte professionnel. Puis nous cherchons à souligner l'apport des notions de capital social et de réseaux sociaux pour le rôle de l'acteur commercial. Cela nous conduit à voir les réseaux sociaux comme un outil intuitivement employé depuis longtemps par ces professionnels, et à poser un certain nombre de questions à visée opérationnelle : comment nait puis est utilisé son carnet d'adresses ? Cherche-t-il à formaliser son approche relationnelle des affaires ? Quelle part fait-il entre ses relations privées et ses liens professionnels, dans le marché ou l'entreprise ? Etc.

Finalement, nous présentons la démarche exploratoire que nous entamons actuellement, dans le cadre d'un programme de recherche plus vaste portant sur le capital social des membres de la force de vente. Une série d'entretiens en profondeur réalisée en 2007 a précédé une enquête qui vise plus de 2000 managers et négociateurs commerciaux. Nous cherchons notamment à déterminer comment des négociateurs commerciaux constituent et utilisent leurs réseaux sociaux, comment ils formalisent cette démarche au sein d'un groupe constitué de leurs pairs, et quels bénéfices ils en tirent pour leur activité quotidienne.

\section{Le contexte de la vente comme processus social dynamique.}

De très nombreux métiers ayant adopté le nom de «vendeur » ou de «négociateur », il faut définir le métier de négociateur commercial au sens où nous l'entendons, avant d'en examiner le contexte spécifique et de justifier l'intérêt d'une telle recherche.

\subsection{Le négociateur commercial : définition.}

La complexité de la vente conduit aujourd'hui ses acteurs à être en relation avec de multiples interlocuteurs (Audebert-Larochas, 2004), ce qui prend un sens plus fort pour le négociateur commercial, "appelé parfois ingénieur d'affaires [qui] se distingue du représentant (...) ou du technico-commercial par la qualité et l'étendue de ses connaissances » (Jolibert et Tixier, 1988, p. 9). Outre l'aspect cognitif, la nature des relations distingue le négociateur commercial du vendeur : l'acheteur et l'offreur peuvent tous deux fixer et faire évoluer le cadre de leurs relations (Jolibert et Tixier, 1988).

Parmi diverses catégories possibles, nous parlons notamment ici des ingénieurs commerciaux et des managers de comptes-clés (Brassier, 2006). Nous ajoutons les cadres dirigeants s'ils conservent une forte part d'activité commerciale, orientée vers les plus grands comptes. Le poids des affaires qu'ils négocient et leur impact individuel sur les résultats de l'entreprise 
sont considérables. Ils sont très autonomes, ont le mandat pour décider, et sont issus de l'enseignement supérieur. Ils nouent pour leur organisation des partenariats stratégiques.

Auparavant concentré sur la création de liens unipersonnels avec ses interlocuteurs, le rôle du négociateur commercial est aujourd'hui celui d'une interface dans une relation complexe, composée d'étapes et d'interlocuteurs multiples, dont la portée et la durée sont très éloignées d'un «évènement discret » (Dwyer et alii, 1987). Lien privilégié entre l'organisation et son marché, il crée de la valeur ajoutée pour une grappe de partenaires interdépendants (Anderson, 1996). C'est encore plus vrai dans le cas de négociateurs commerciaux internationaux : la mondialisation en fait des pivots entre l'entreprise et ses grands clients (Wilson et Millman, 2003). De ce fait, les compétences dorénavant requises des négociateurs commerciaux vont bien au-delà de la vision traditionnelle (Wotruba, 1995), et sont plus souvent d'ordre stratégique qu'opérationnel, en particulier concernant la gestion de leur portefeuille de contacts (Lambe et Spekman, 1997). Leur connaissance des partenaires doit être approfondie pour permettre de déceler les situations intéressantes, autant que doit l'être la connaissance de leur propre organisation pour évaluer son potentiel de réponse aux besoins identifiés. Leurs savoir-faire englobent la créativité dans la résolution de problèmes complexes, l'interaction interculturelle, le management des conflits, la création de confiance, le management de projets, le travail collaboratif ou encore le leadership (Weitz et Bradford, 1999). Ils doivent en fait avoir des capacités d'entrepreneurs internes (Morris, Avila et Teeple, 1990) dans une approche de marketing relationnel.

Le négociateur commercial est situé à un poste d'intérêt stratégique pour son entreprise, faisant de ses réseaux sociaux une ressource cruciale pour la conduite de ses affaires, procurant un avantage organisationnel peu mis en valeur et exploité (Nahapiet et Ghoshal, 1998). Tout particulièrement en situation de changement permanent, propre à l'économie moderne, il est l'un des maillons permettant à l'organisation d'évoluer, du fait de ses liens internes et externes, et des caractéristiques de sa fonction. En effet, les interactions entre équipes sont l'un des moyens privilégiés de diffuser de nouveaux savoirs aux acteurs organisationnels, et de développer leurs nouveaux schèmes de pensée et de comportement. $\mathrm{Au}$ lieu d'une vision hiérarchique, les relations informelles entretenues par certains acteurs, dont les commerciaux, avec différents services, favorisent l'échange et le partage d'information et de points de vue (Albers Mohrman, Tenkasi et Mohrman Jr., 2003, McGrath et Krackhardt, 2003). Les réseaux sociaux sont des outils de gestion des liens intra-organisationnels et interorganisationnels à multiniveaux (Brass et alii, 2004).

\subsection{Le marketing relationnel.}

Une relation commerciale vaut par la qualité de ses partenariats et de leurs processus sociaux (Levy et Zaltman, 1975, Kaufman et Stern, 1988, Weitz et Bradford, 1999), ce que met en avant le marketing relationnel (Bagozzi, 1975), dans un contexte de concurrence accrue, de marchés mondialisés, et d'acteurs moins nombreux mais plus importants et plus interconnectés qu'auparavant. Bien au-delà de la transaction, "le client [doit être] au centre des préoccupations internes» des entreprises, par le «développement de la confiance entre acteurs internes et externes de la relation 'vente' ", et par "la profitabilité croissante des relations avec les clients »(Bergadaà et Laaroussi, 2005, p. 3).

Le marketing relationnel est devenu dans les années 90 le nouveau paradigme du marketing. Le rôle partenarial du commercial devient nécessaire : peser sur l'acte d'achat est dépassé, l'on mise dorénavant sur la gestion des conflits inhérents à la relation à long terme entre les parties (Weitz et Bradford, 1999). Mais au-delà de son seul lien vendeur-acheteur, le négociateur est l'acteur multipolaire situé au cœur d'un monde d'information constitué de réseaux interconnectés, garant de la fluidité des échanges qu'il initie (Gensollen, 2001). Il est 
l'un des maillons incontournables de l'entreprise qui navigue parmi ces interconnections formelles et informelles, et dont la politique marketing doit intégrer à la fois le long terme et la multiplicité des relations avec ses clients.

D'autres acteurs sont concernés par cette approche relationnelle du marketing, d'où une coordination accrue interservices, dans le cadre d'un développement commercial multicanal, pour un meilleur lien entre le marketing stratégique et la vente opérationnelle. Si le réseau commercial maille le territoire d'une manière cohérente (Fournier, 2004), chaque client peut obtenir la même information, fiable, complète et adaptée, et l'entreprise reçoit en retour une information pertinente sur sa relation au marché, en cumulant et/ou en croisant les informations provenant des différents points de son circuit commercial. Cependant, cette vision structurelle du réseau commercial manque souvent d'une conception relationnelle du métier des acteurs commerciaux, au premier rang desquels se situe le négociateur commercial.

\section{Le négociateur commercial, acteur social par essence.}

Depuis une dizaine d'années l'on voit que les réseaux sociaux sont des ressources d'action au service du négociateur commercial (Degenne et Forsé, 1994, Adler et Kwon, 2002, Huault, 2002). Ces ressources constituent le capital social du négociateur, qui est l'ensemble des relations tissées au cours d'actions collectives, au sein desquelles circulent confiance, normes partagées, inter-reconnaissance et possibilités d'échange (Lecoutre, 2006). Tant du point de vue de la forme que du contenu, ce capital est d'un intérêt vital pour le négociateur professionnel, en tant que ressource relationnelle pour l'action (Brassier et Lecoutre, 2007).

\subsection{Les réseaux sociaux, ressource pour la négociation commerciale.}

Concernant la forme du réseau, la négociation bénéficiera d'apports informationnels inédits grâce aux liens établis en dehors de leurs cercles habituels, ce que l'on nomme des liens faibles (Granovetter, 1973, 1995). Cela dépend cependant du contexte : on peut vouloir utiliser des trous structuraux pour bénéficier de liens inédits, pour prospecter de nouveaux partenaires par exemple. Un réseau large et diversifié est propice au démarrage de relations d'affaires, tandis qu'un maillage constitué d'une poignée de liens sociaux forts importe tout particulièrement pour parvenir à un accord dans une affaire (Üstuner et Godes, 2006).

Concernant le contenu du capital social, il apparait que les négociateurs privilégient la collaboration, voire l'encastrement social dans leur activité. Mais les parts instrumentale et non instrumentale de leurs relations restent mêlées (Morgan et Hunt, 1994, Wilson, 1995, Granovetter, 2002, Steiner, 2002, Jones et McCleary, 2004). Une négociation visant un avantage partagé et pérenne se construira donc sur des principes d'échanges tant identitaires qu'utilitaires (Lecoutre et Lièvre, 2005, Lièvre et Lecoutre, 2006). Le rapprochement identitaire, la densité des contacts (Harris, O’Malley et Patterson, 2003) ou l'homogénéité des valeurs constitueront dès lors des compétences pour un négociateur au même titre que ses savoir-faire techniques.

Par son maillage social, le négociateur obtient un accès privilégié à des informations et à des décisions de premier plan. Si le marketing est relationnel, alors les relations interindividuelles sont un moyen de le matérialiser (Iacobucci, 1996). C'est au niveau individuel qu'il importe désormais de faire ressortir les rapports entre les affaires réalisées et les contacts personnels (Gulati, 1995). Créer une relation commerciale signifie selon les cas transformer une relation existante en partenariat d'affaires, ou envisager d'emblée ses relations avec une visée pragmatique, pour de futurs succès économiques (Dacin et alii, 1999).

Le capital social facilite l'échange de ressources, la création du capital intellectuel, l'efficacité des équipes, l'entrepreneuriat, ainsi que les relations clients-fournisseurs (Adler et Kwon, 
2002). L'intuition-clé est l'a priori positif à l'égard de l'individu, utilisable comme une ressource de grande valeur. On voit bien qu'un négociateur peut profiter d'une position qui l'amène à être soit un «pont» entre les groupes dans l'organisation, avec d'autres commerciaux, et avec divers partenaires externes, soit un «gardien » de la relation intragroupe, avec ses relations professionnelles habituelles. L'adhésion à des associations professionnelles, dite «affiliation institutionnelle » est d'ailleurs une forme intéressante de liens externes (Belliveau, O’Reilly et Wade, 1996).

En investissant les trous structuraux, le négociateur devient un intermédiaire dont a besoin son employeur, avec son marché mais aussi entre ses services internes. Il a la main sur des liens rares procurant des ressources critiques (Burt 1992, 1995). L'entreprise est fortement tenue par ces liens et par leur détenteur, qu'un contexte d'affaires mouvant rend relativement unique. Aucune routine rigide ni standard formel n'est transmissible en l'état (Blyler et Coff, 2003).

La centralité du négociateur lui donne également un pouvoir accru (Ibarra, 1993). Il a une certaine maitrise des turbulences des marchés, grâce à ses connexions avec de nombreux partenaires et contacts. Il produit en fait de la stabilité économique et sociale pour l'ensemble des acteurs en jeu. Il devient un distributeur d'information cruciale, un relais de décision ou un répartiteur de ressources. Il peut agir souplement «entre la force de l'autorité organisationnelle et la dextérité des marchés », connectant les éléments dispersés de l'entreprise et de son environnement (Burt, 1998). Il est l'entremetteur entre des groupes et des individus, le gardien du pont dont dépendent les habitants des deux rives pour leur commerce.

Sa valeur ajoutée consiste justement à être ce passeur chargé de négocier avec de multiples acteurs, sans autorité hiérarchique sur leurs demandes parfois contradictoires, avec une vision stratégique. Il agit en entrepreneur, de ce fait : le négociateur se révèle être un expert en gestion des trous structurels, acteur souple s'affranchissant des contraintes de la structure hiérarchique. Pour cela, il valorise l'information et les décisions prises, pour des propositions ou des accords notamment, en réorganisant continuellement ses ressources pour imaginer des solutions inédites (Burt, 1998). A l'aide de son capital social et de l'expérience acquise, l'entrepreneur qu'il est a ainsi pour tâche de reconnaitre et de saisir les opportunités du marché. Il a donc logiquement pour ce faire une vision très personnelle des risques encourus, des chances de succès, de ses propres capacités de réussite, ou encore des intérêts personnels et collectifs à considérer (De Carolis et Saparito, 2006).

\subsection{Le négociateur, vecteur d'encastrement social pour l'entreprise.}

La négociation commerciale bâtit ses objectifs à long terme sur un partenariat tissé de relations personnelles imbriquées, quel que soit le secteur d'activité (Wilson, 1995, Wotruba, 1991). Les commerciaux construisent, maintiennent et optimisent ainsi « les relations proches et coopératives avec un ensemble limité de fournisseurs, de clients et de membres du canal » commercial (Weitz et Bradford, 1999, p. 241). Par ses contacts internes, le négociateur mobilise des ressources complémentaires nécessaires à une meilleure gestion du partenariat (Wall et Callister, 1995, Johnston et Marshall, 2003). Dans ce sens, l'acheteur reporte sur le négociateur une part croissante de responsabilité pour valoriser leur partenariat.

Bien que les entreprises structurent et formalisent la gestion des clients comptes-clés pour obtenir une valeur partagée nettement améliorée, ces derniers ne perçoivent pas clairement en quoi leurs fournisseurs créent de la valeur par une approche plus relationnelle (Ivens et Pardo, 2004). La question de l'impact de la vente relationnelle est alors posée. Quel type de relation le négociateur construit-il avec les clients ? Comment encourage-t-il l'échange dans ses relations ? Quels modes de travail et de contrôle sont activés ? (McKenzie, 2001). Certes, au 
plan général, le commercial devient garant d'échanges et de liens efficaces, procurant de la valeur pour l'ensemble des parties engagées, à court et à long terme (Anderson et Dubinsky, 2004). Quelques acheteurs importants prennent désormais une grande valeur commerciale, ce qui est le propre des comptes-clés, dont l'entreprise veut pouvoir mieux prédire le comportement, afin d'engager des relations pérennes en mobilisant des ressources mutualisées (Manning et Reece, 2007). Pour la résolution de problèmes communs, il établit des liens multiples chez le client comme dans son entreprise, ou développe la confiance avec son client tant au plan individuel qu'organisationnel (Day, 2000).

Ceci dit, pour être effectivement relationnel, le marketing ne peut plus se penser uniquement au plan inter-organisationnel : chaque négociateur établit ses contacts avec une foule d'acteurs possédant des ressources propres à l'aider dans l'atteinte durable de ses objectifs. Il sait que le client travaille également son réseau de contacts pour rendre ses achats plus sûrs (Katz et Lazarsfeld, 1955). Coopérer uniquement avec l'acheteur est insuffisant ; la relation d'affaires implique divers acteurs complémentaires, dans les services de l'entreprise comme dans les contacts externes (Yilmaz et Hunt, 2001). On peut parler à la fois de liens structurels orientés vers un résultat commercial et de liens sociaux basés sur la confiance et la satisfaction interpersonnels (Jones et McCleary, 2004, Williams, Han et Qualls, 1998). L'attractivité commerciale d'un partenaire dépendra de la perception que son interlocuteur aura de sa capacité à fournir des gains économiques, des accès à des ressources ou de leur compatibilité sociale (Harris, O’Malley et Patterson, 2003). La relation économique est optimisable grâce à une action de socialisation basée sur les valeurs communes, l'engagement et la confiance qui en découle (Husted, 1994), sur la proximité et la convivialité (opportunités d'interactions, tels que des évènements sociaux), ou encore sur le renouvellement fréquent des rencontres (Harris, O’Malley et Patterson, 2003).

Du fait de la nature dynamique de ces relations commerciales, le négociateur s'appuie sur ses capacités relationnelles (Dubinsky et alii, 1986), sur l'apprentissage partagé et le travail collaboratif, en vue du résultat commun (Moon et Armstrong, 1994). Il étend ses relations hors des frontières organisationnelles pour accroitre son pouvoir au moyen des informations privilégiées qu'il obtient (Manev et Stevenson, 2001). Finalement, négocier c'est engendrer un processus social dynamique grâce aux relations individuelles, aux valeurs et aux cadres de pensée communs, et à une approche tant identitaire qu'utilitariste (Jönsson, 2005). Il faut pour cela établir des liens variés avec les acteurs de l'entreprise et ceux du marché, dont la force influencera les phases de la négociation (Üstuner et Godes, 2006). Partant de visites informelles, la stratégie relationnelle du commercial se développera jusqu'à ce qu'il devienne idéalement un partenaire incontournable (Darr, 2007).

\section{Etude exploratoire des réseaux sociaux du négociateur commercial.}

Notre approche est innovante par rapport aux précédents travaux sur les réseaux sociaux pour au moins deux raisons : l'une est qu'il s'agit d'un travail de gestion, et non d'une sociologie appliquée aux acteurs économiques. Notre but à terme est par conséquent de montrer en quoi le capital social du négociateur est effectivement un outil de gestion (Berry, 1983). La seconde raison est que nous voulons étudier le capital social du négociateur commercial dans son évolution : comment il constitue, développe et maintient ses ressources relationnelles, quelle nature et quelle forme a son réseau, quels acteurs le constituent, quels contenus il cherche et parvient à développer. Nous voulons savoir si ces relations sont des tractations uniquement commerciales, ou si elles participent à un développement identitaire, basé sur l'appartenance à un même groupe. Une relation sociale peut-elle devenir commerciale, et inversement ? Comment se fonde la coopération ? Quelles conditions permettent à une relation de devenir un partenariat commercial durable? 
Il s'agit, dans un premier temps, d'une recherche qualitative exploratoire, en partie descriptive (Ghauri et alii, 1995, Wacheux, 1996). Des interviews en profondeur nous livrent des éléments complexes et détaillés d'information concernant les pratiques des négociateurs (Demers, 2003). Elles sont menées à l'aide d'un guide semi-directif, construit d'après une revue de la littérature et notre expérience du domaine de la négociation commerciale. Les entretiens sont enregistrés au magnétophone et intégralement retranscris par écrit, des notes manuscrites prises en cours d'interview complétant le relevé d'information. L'entretien porte sur le contexte de l'activité professionnelle, pour situer l'importance en valeur comme en volume des contacts d'affaires, puis sur la manière de créer et de gérer ces contacts. Le but est de mieux cerner le nombre et la variété des acteurs appartenant au réseau relationnel de l'interrogé, l'évolution du capital relationnel dans la durée, et s'il existe une stratégie particulière de prise de contact et de maintien du capital social.

Les 20 à 30 entrevues programmées tiennent notamment compte de la diversité des secteurs d'activité et des régions, de l'expérience, et de l'équilibre entre hommes et femmes, qui est d'ailleurs, dans les positions de management commercial, très éloigné de ce qu'il est dans la population française. Nous livrons ici une synthèse des huit premières interviews réalisées, avant de réaliser sur l'ensemble une analyse thématique de contenu (Jolibert et Jourdan, 2006). Les catégories prédéterminées s'appuient pour une part sur la littérature existante en réseaux sociaux: taille du capital social, types d'acteurs, liens faibles/forts, ressources échangées, etc. Pour une autre part, les questions novatrices auxquelles nous voulons répondre permettre de proposer des catégories inédites : temps investi, durée du capital social, actions clés, stratégie activée, etc. Notre démarche, après ce premier stade, suivra les critères d'évaluation d'une recherche qualitative permettant de considérer nos résultats comme crédibles, confirmables, contextualisés et transférables (Lincoln et Guba, 1986).

\subsection{Le groupe de négociateurs commerciaux mobilisé.}

Les interviews réalisées montrent la diversité des contextes rencontrés, tout en rassemblant des points communs particulièrement intéressants. Bien que confirmant certains points déjà connus dans la littérature, elles apportent des éléments nouveaux significatifs.

\begin{tabular}{|c|c|c|c|c|c|c|c|}
\hline Interview & Région & Genre & Secteur & Poste & Expérience & Formation & Clients \\
\hline $\mathrm{N}^{\circ} 1$ & Paris & Homme & Banque patrimoniale & $\begin{array}{l}\text { Conseiller } \\
\text { commercial }\end{array}$ & 1,5 an & $\begin{array}{l}\text { Scientifique, } \\
\text { économique }\end{array}$ & $\begin{array}{l}\text { Particuliers, } \\
\text { Gros } \\
\text { patrimoines }\end{array}$ \\
\hline $\mathrm{N}^{\circ} 2$ & Clermont & Homme & $\begin{array}{l}\text { Industrie } \\
\text { Electrique }\end{array}$ & $\begin{array}{l}\text { Ingénieur } \\
\text { commercial }\end{array}$ & 15 ans & $\begin{array}{l}\text { Commerce, } \\
\text { Mécanique }\end{array}$ & $\begin{array}{l}\text { Entreprises, } \\
\text { Collectivités }\end{array}$ \\
\hline $\mathrm{N}^{\circ} 3$ & Bordeaux & Femme & Presse & $\begin{array}{l}\text { Associée } \\
\text { PME }\end{array}$ & 13 & Communication & Entreprises \\
\hline $\mathrm{N}^{\circ} 4$ & Colmar & Homme & Banque de détail & $\begin{array}{l}\text { Directeur } \\
\text { d'agence }\end{array}$ & 18 & Gestion & $\begin{array}{l}\text { Particuliers, } \\
\text { PME }\end{array}$ \\
\hline $\mathrm{N}^{\circ} 5$ & Toulouse & Femme & Télécommunications & $\begin{array}{l}\text { Ingénieur } \\
\text { commercial }\end{array}$ & 10 & Gestion & $\begin{array}{l}\text { Collectivités } \\
\text { publiques }\end{array}$ \\
\hline $\mathrm{N}^{\circ} 6$ & Nantes & Homme & Conseil & Consultant & 6 & $\begin{array}{l}\text { Marketing, } \\
\text { commerce }\end{array}$ & $\begin{array}{l}\text { Entreprises, } \\
\text { Collectivités }\end{array}$ \\
\hline $\mathrm{N}^{\circ} 7$ & Marseille & Homme & Télécommunications & $\begin{array}{l}\text { Directeur } \\
\text { régional }\end{array}$ & 10 & Commerce & Distributeurs \\
\hline $\mathrm{N}^{\circ} 8$ & Nice & Homme & $\begin{array}{l}\text { Technologie } \\
\text { d'information }\end{array}$ & Consultant & 4 & Informatique & Entreprises \\
\hline
\end{tabular}

La plupart des interviewés ont hérité de portefeuilles de contacts structurés par leurs prédécesseurs. Les indépendants $\left(\mathrm{N}^{\circ} 2,6\right.$ et 8$)$ sont plus démunis sur ce plan, et tâchent de s'appuyer sur leur propres acquis. Tous sont très actifs relationnellement, et souvent adhérent à plusieurs réseaux formels, dans le cadre de leur fonction ou à titre personnel. Leur métier les 
a tous amenés à gérer des clients de taille et de complexité croissantes, que ce soit dans le privé ou le public, en vente directe ou par intermédiaires. Leur choix de carrière est guidé par le goût de l'indépendance, d'un métier riche en diversité, de l'engagement avec des partenaires. Ils sont soit initialement formés, soit ont développé des connaissances poussées en marketing, commerce, vente, voire en sens relationnel et politique. Leur vision commune les conduit véritablement à investir leurs efforts et leur temps pour parvenir à terme à une réussite économique solide. Les non indépendants sont enclins à le devenir (non mentionné par 4 et 7$)$. Les femmes $\left(\mathrm{N}^{\circ} 3\right.$ et 5$)$ disent devoir beaucoup plus «prouver leur position ${ }^{2} »$ et leurs compétences que les hommes avant d'être reconnues comme telles.

\subsection{Les stratégies de constitution des réseaux sociaux des négociateurs.}

Un relatif consensus existe sur la création des réseaux sociaux par les négociateurs. Ils constituent leur réseau par des moyens que nous pourrions qualifier d'heuristiques: les contacts déjà acquis, les clients, les prescripteurs, la recommandation, le bouche à oreille, les amis éventuellement, les occasions conviviales (dîners, salons professionnels, etc.), ou « au fil de l'eau ». Générer des relations c'est «fréquenter un endroit qui va bien », et utiliser en parallèle quelques réseaux. Tous pensent que prospecter est une perte de temps, et dérange des professionnels eux-mêmes trop visités, ce qui rend plus difficile encore le premier contact. Une fois établi, et y compris si l'on traite avec un compte-clé, la relation doit devenir proche et familière, au sens de Harris et alii (2003).

C'est par conséquent en «douceur » que les contacts seront approchés, éventuellement grâce à un intermédiaire, permettant souvent un gain de temps dans l'approche délicate de prospects qui doivent sélectionner de plus en plus leurs interlocuteurs. Ils seront «sensibles » à la subtilité avec laquelle on les abordera, même dans un contexte a priori destiné au relationnel d'affaires. Plutôt qu'une stratégie de prospection systématique, on privilégiera l'attention portée au potentiel de chaque contact établi, qu'il soit amical, lié à la vie sociale ou dans le cadre du travail.

Les négociateurs mettent en avant l'importance, tant en impact qu'en investissement de temps, de "découvrir l'autre ", autrement dit d'élaborer une relation interpersonnelle avant d'afficher ses objectifs professionnels. Plus les contacts semblent détenir un fort potentiel, être «politiques », plus ils doivent être «chouchoutés » et pris en considération dans cette approche en deux temps. Cela justifie d'être très investi sur «le terrain », pour être reconnu, et pour pouvoir à la fois « sélectionner » les relations que l'on veut initier et attendre « le bon moment», «ne pas forcer» la rencontre. Il y a une forme de rituel dans ses approches progressives des «chaines de décision» propres aux clients clés, qui se dévoilent dans la durée seulement, mais où chacun pourrait avoir une influence qu'il faut découvrir.

Conséquemment, c'est sur la confiance réciproque que se bâtissent des relations qui durent souvent au moins une décennie. C'est si l'on est perçu comme un interlocuteur personnellement et professionnellement «fiable » que l'on s'assure de la pérennité de cet investissement relationnel, dans lequel l'image de marque est cruciale, et doit être entretenue au moindre signal, qu'il indique une affaire potentiel ou un risque à venir.

\subsection{La structure des réseaux sociaux développés.}

Les types de contacts sont très variés, généralement. Le négociateur sélectionne ses contacts, tout en s'accommodant de leur variété : il cherche à «dénicher » ceux qui peuvent lui ouvrir

\footnotetext{
${ }^{2}$ Dans cette partie du texte, les expressions placées entre guillemets sont extraites du verbatim. Plutôt que des paragraphes entiers, une sélection de mots les plus illustratifs de perceptions saillantes a été retenue.
} 
« un bon carnet d'adresses », qui seront ses « relais» pour être «coopté » par de réels comptes clés, mais aussi pour qu'il construise sa réputation localement. Pour ce faire il tend à cibler des dirigeants, des personnalités diverses (comptables, élus, etc.), des décideurs publics, des cadres associatifs, etc. l'un de ses premiers objectifs de développement du capital relationnel consiste ainsi à se faire admettre par les «prescripteurs importants ».

Selon le contexte, ce capital social représente alors de quelques dizaines de contacts $\left(\mathrm{N}^{\circ} 8\right)$ à un millier $\left(\mathrm{N}^{\circ} 5\right)$, parmi lesquels existent seulement « quelques liens forts » qui assureront un relais de contact auprès d'autres personnes, et qui peuvent aussi être les alliés en qui l'on aura le plus confiance. Néanmoins, un nombre conséquent de contacts est nécessaire pour déceler, au fil du temps, ceux qui possèdent une position de trou structurel donnant accès à une valeur ajoutée certaine.

En gestionnaire, le négociateur estime qu'il investi ses efforts à constituer ainsi son capital social, qui nécessite un minimum de deux années $\left(\mathrm{N}^{\circ} 2\right.$ et 5$)$ pour qu'il commence à être fructueux. Et d'investissement il s'agit puisque, surtout pour les liens les plus serrés, la relation peut durer une vingtaine d'années semble-t-il. Cela tient compte, naturellement, de la carrière du négociateur, qui peut évoluer dans d'autres entreprises, d'autres secteurs d'activité ou d'autres régions, et par là-même se couper de ses contacts patiemment établis.

\subsection{Les dimensions utilitaires et sociales des réseaux sociaux du négociateur.}

La relation n'est pas constituée que de «business », certes «assumé, visible », mais qui matérialise une dimension instrumentale secondaire dans la relation sociale, pour tous les cas rencontrés. Des notions d'entraide, de solution partagée sur des problèmes identiques, d'état d'esprit et de valeur identitaires, d'apprentissage dans l'échange, de «don de soi », de «conseils pratiques », importent aux négociateurs dans leurs relations, aussi bien pour en bénéficier que pour en procurer. Tout en ne perdant pas de vue l'intérêt économique de la relation, les signes de don sont un préalable à la construction d'une réputation professionnelle fiable. Comme ils souhaitent trouver des ressources de nature non économique dans leur réseau social, il est cohérent de montrer que l'on a la capacité et l'attitude susceptibles d'être soi-même une ressource pour d'autres interlocuteurs qui n'auraient pas naturellement pensé à eux.

En définitive, un capital social est une ressource qui mérite des efforts conséquents et durables de la part des commerciaux. Un contact acquis ne révèlera son potentiel caché qu'au fil du temps. Cela ne peut se faire par les moyens classiques de prospection commerciale. Le négociateur doit être disponible, «réceptif » aux signes interpersonnels et sociaux, ouvert à une ritualisation de la relation plus ou moins formalisée, que ce soit pour chaque nouvelle rencontre ou parmi ses réseaux affiliés.

\section{Conclusion.}

La négociation est une activité réticulaire par essence et par nécessité. Cette donnée doit être formellement intégrée dans les compétences indispensables au négociateur. Les objectifs qu'il poursuit avec des partenaires de grande taille, qui recherchent un degré de confiance élevé pour collaborer, en dépendent. Le réseau social est alors un outil crucial pour mieux probabiliser le succès socio-économique et réduire les incertitudes d'une relation d'affaires.

Notre recherche, qui n'en est qu'à son début, souligne la place de ce type de compétence, de la valeur des informations, ou de l'investissement de temps indispensable dans les échanges interpersonnels propres à l'exercice de la négociation commerciale. Au plan managérial, elle vise à analyser la nature, la forme, l'efficacité du réseau relationnel en tant que ressource d'action en négociation, ce qui n'a jamais été entrepris. Plus généralement, tout un pan de 
recherche peut s'ouvrir à présent sur le capital social des individus et des entreprises comme outil de management : quelles seraient les «bonnes » méthodes de mobilisation du réseau social si l'on considère qu'il est un outil de gestion à part entière ? Comment passer de pratiques largement non formalisées et intuitives, à des processus plus intentionnels et formalisés, transférables dans l'organisation ? Ce sont quelques-uns des points que nos travaux visent à éclaircir.

\section{Bibliographie.}

P. S. Adler et S.-W. Kwon (2002), «Social Capital: Prospects for a New Concept », Academy of Management Review, 27(1), pp. 17-40.

S.Albers Mohrman, R. V. Tenkasi et Mohrman Jr. A. M. (2003), «The Role of Networks in Fundamental Organizational Change: A Grounded Analysis », Journal of Applied Behavioral Science, 39, pp. 301-323.

R. E. Anderson (1996), «Personal Selling and Sales Management in the New Millennium », Journal of Personal Selling \& Sales Management, 16(4), pp. 17-32.

R. E. Anderson et A. J. Dubinsky (2004), Personal Selling: Achieving Customer Satisfaction and Loyalty, Houghton Mifflin, Boston.

P. Audebert-Larochas (2004), «Comment différencier négociation et vente », in La négociation, Revue Française de Gestion, 153, pp. 141-155.

R.P. Bagozzi (1975), « Marketing as Exchange », Journal of Marketing, 39, pp. 32-39.

M. A.Belliveau, C. A. O’Reilly et J. B. Wade (1996), «Social Capital at the Top: Effects of Social Similarity and Status on CEO Compensation », Academy of Management Journal, 39, pp. 1568-1593.

M. Bergadaà et S. Laaroussi (2005), « Mythes et réalités de la mutation d'un métier : le point de vue de la vente dans le B to B », Association Française de Marketing, Saint-Malo, 18 p.

M. Berry (1983), «L'impact des instruments de gestion sur l'évolution des systèmes humains », CRG, Paris, Juin, $51 \mathrm{p}$.

M. Blyler et R. W. Coff (2003), « Dynamic Capabilities, Social Capital and Rent Appropriation: Ties that Split Pies », Strategic Management Journal, 24, pp. 677-686.

D. J. Brass, J. Galaszkiewicz, H. R. Greve et W. Tsai (2004), “Taking Stock of Networks and Organizations: A Multilevel Perspective”, Academy of Management Journal, 47(6), pp. 795-817.

P. Brassier (2006), Qui sont les commerciaux?, Editialis-Action Commerciale, Paris.

P. Brassier et M. Lecoutre (2007), «Réseaux sociaux et négociateurs d'affaires : étude empirique d'un facteur discriminant pour la gestion du risque dans les négociations commerciales », $3^{e}$ Biennale Internationale de la Négociation, Négocia, Paris, 23 p.

R. S. Burt (1992), Structural Holes. The Social Structure of Competition, Harvard University Press, Cambridge, Mass. et Londres.

R. S. Burt (1995), «Le capital social, les trous structuraux et l'entrepreneur », Revue Française de Sociologie, 36(4), pp. 599-628.

R. S. Burt (1998), « The Gender of Social Capital », Rationality and Society, 10(1), pp. 5-46.

M. T. Dacin, M. J. Ventresca et B. D. Beal (1999), «The Embeddedness of Organizations: Dialogue \& Directions », Journal of Management, 25(3), pp. 317-356.

A. Darr (2007), «The Mutual Weaving of Obligation Networks in Mass Industrial Markets », Current Sociology, 55(1), pp. 41-58.

G. S. Day (2000), « Managing Market Relationships », Journal of the Academy of Marketing Science, 28(1), pp. 24-30.

D. M. De Carolis et P. Saparito (2006), « Social Capital, Cognition, and Entrepreneurial Opportunities: A

Theoretical Framework », Entrepreneurship Theory and Practice, Janvier, pp. 41-56.

A. Degenne et M. Forsé (1994), Les réseaux sociaux, $1^{\text {ère } e ́ d, ~ A r m a n d ~ C o l i n, ~ P a r i s . ~}$

C. Demers (2003), «L'entretien », in Giordano Y. (Dir.) Conduire un projet de recherche, une perspective qualitative, EMS, Paris, pp. 173-210.

A. J. Dubinsky, R. D. Howell, T. N. Ingram et D. Bellenger (1986), « Sales Force Socialization », Journal of Marketing, 50(4), pp. 192-207.

F. R. Dwyer, P. H. Shurr et S. Oh (1987), «Developing Buyer-Seller Relationships », Journal of Marketing, 51(2), pp. 11-27.

C. Fournier, «L'impact d'une stratégie multicanale sur la force de vente : l'émergence du client "communautaire", Colloque Le Management Click and Mortar, Nice, 18 p.

M. Gensollen (2001), «Internet. Marché électronique ou réseaux commerciaux ? », Revue économique, 52(7), pp. 137-161.

P. Ghauri, K. Grønhaug et I. Kristianslud (1995), Research Methods in Business Studies, Prentice-Hall, Londres. 
M. S. Granovetter (1973), «The Strength of Weak Ties », American Journal of Sociology, 78, pp. 1360-1380.

M. S. Granovetter (1995), Getting a Job. A Study of Contacts and Careers, $2^{\text {nd }}$ ed., (1 ${ }^{\text {st }}$ ed. 1974), Harvard University Press, Cambridge, Mass. \& London.

M. S. Granovetter (2002), «Préface », in Huault I. (Dir.), La construction sociale de l'entreprise, EMS, Paris, pp. 9-14.

R Gulati (1995), « Does Familiarity Breed Trust? The Implications of Repeated Ties for Contractual Choice in

Alliances ». Academy of Management Journal, 38, pp. 85-112.

L. C. Harris, L. O’Malley et M. Patterson (2003), «Professional Interaction: Exploring the Concept of Attraction », Marketing Theory, 3(1), pp. 9-36

I. Huault (Dir.) (2002), La construction sociale de l'entreprise. Autour des travaux de Mark Granovetter, EMS, Paris.

B. W. Husted (1994), «Transaction Costs, Norms, and Social Networks: A Preliminary Study of Cooperation in Industrial Buyer-Seller Relationships in the United States and Mexico », Business Society, 33, pp. 30-57.

D. Iacobucci (1996), Networks in Management, Sage, Londres.

H. Ibarra (1993), « Network Centrality, Power, and Innovation Involvement: Determinants of Technical and

Administrative Roles », Academy of Management Journal, 36(3), pp. 471-502.

B. S. Ivens et C. Pardo (2004), «Les clients comptes clés sont-ils vraiment traités différemment ? Le point de vue des clients », Recherche et Applications en Marketing, 19(4), 20 p.

D. L. Jones et K. W. McCleary (2004), « A Model for Assessing Cultural Impacts on International Buyer-Seller Relationships for Key Accounts of Hotel Companies”, Journal of Hospitality and Tourism Research, 28, pp. 425-443.

M. W. Johnston et G. W. Marshall (2003), Churchill/Ford/Walker's Sales Force Management, $7^{\text {th }}$ Edition, McGraw-Hill, New-York, N.Y.

A. Jolibert et P. Jourdan (2006), Marketing Research. Méthodes de recherche et d'études en marketing, Dunod, Paris.

A. Jolibert et M. Tixier (1988), La négociation commerciale, Etudes de cas, préparation et stratégie, ESF, Paris.

C. Jönsson (2005), «Developing Social Relations: A Neglected Aspect of Negotiations », 2e Biennale Internationale de la Négociation, Négocia, Paris, 13 p.

E. Katz et P. F. Lazarsfeld (1955), Personal Influence, Free Press, Glencoe, Il.

P. J. Kaufmann et L. W. Stern (1988), « Relational Exchange Norms, Perceptions of Unfairness, and Retained Hostility in Commercial Litigation », Journal of Conflict Resolution, 32, pp. 534-552.

C. J. Lambe et R. Spekman (1997), « National Account Management: Large Account Selling or Buyer-Seller Alliance? », Journal of Personal Selling and Sales Management, 17(3), pp. 61-74.

M. Lecoutre (2006), « Le capital social dans les transitions écoles-entreprises », in Bevort A. et Lallement M. (Dir.), Le capital social. Performance, équité et réciprocité, La Découverte, Bibliothèque du Mauss, Paris, pp. 177-192.

M. Lecoutre et P. Lièvre (2005), «Le lien faible "potentiellement coopératif": l'amorce du processus de mobilisation des réseaux sociaux dans une démarche de projet », Journée transdisciplinaire AIMS-AGRH Management et réseaux sociaux, EM Lyon.

S. Levy et G. Zaltman (1975), Marketing, Society, and Conflict, Prentice Hall, Englewood Cliffs, N.J.

P. Lièvre et M. Lecoutre (2006), «Le processus de mobilisation des réseaux sociaux dans une démarche de projet: la notion de lien faible potentiellement coopératif. Une application au cas des expéditions polaires », Revue Sciences de Gestion, 52.

Y.S. Lincoln et E.G. Guba (1986), Naturalistic Inquiry, Sage, Thousand Oaks, CA.

I. M. Manev et W. B. Stevenson (2001), «Balancing Ties: Boundary Spanning and Influence in the Organization's Extended Network of Communication », Journal of Business Communication, 38, pp. 183205.

G.L. Manning et B. Reece (2007), Selling Today, Creating Customer Value, Pearson Prentice Hall, Upper Saddle River, N.J.

C. McGrath et D. Krackhardt (2003), « Network Conditions for Organizational Change », Journal of Applied Behavioral Science, 39, pp. 324-336.

R. McKenzie (2001), The Relationship-based Enterprise: Powering Business Success through Customer Relationship Management, McGraw-Hill, New-York, N.Y.

R. M. Morgan et S. D. Hunt (1994), « The Commitment-Trust Theory of Relationship Marketing », Journal of Marketing, 58, pp. 20-38.

M. Morris, R. Avila et E. Teeple (1990), «Sales Management as an Entrepreneurial Activity », Journal of Personal Selling and Sales Management, 10(1), pp. 1-15.

M. A. Moon et G. M. Armstrong (1994), «Selling Teams: A Conceptual Framework and Research Agenda », Journal of Personal Selling and Sales Management, 14(4), pp. 17-30. 
J. Nahapiet et S. Ghoshal (1998), «Social Capital, Intellectual Capital and the Organizational Advantage », Academy of Management Review, 23/2, pp. 242-266.

P. Steiner (2002), «Encastrements et sociologie économique », in Huault I. (Dir.), La construction sociale de l'entreprise, EMS, Paris, pp. 29-50.

T. Üstüner et D. Godes (2006), « Better Sales Networks », Harvard Business Review, 3, pp. 102-112.

F. Wacheux (1996), Méthodes qualitatives et recherche en gestion, Economica, Paris.

J. Wall et R. Callister (1995), « Conflict and Its Management », Journal of Management, 21(3), pp. 515-558.

B. A. Weitz et K. D. Bradford (1999), «Personal Selling and Sales Management: A Relationship Marketing Perspective », Journal of the Academy of Marketing Science, 27(2), pp. 241-254.

J. D. Williams, S. Han, et W. J. Qualls (1998), « A conceptual Model and Study of Crosscultural Business

Relationships », Journal of Business Research, 42, pp. 135-143.

K. Wilson et T. Millman (2003), «The Global Account Manager as Political Entrepreneur », Industrial Marketing Management, 32(2), pp. 151-158.

D. T. Wilson (1995), «An Integrated Model of Buyer-Seller Relationships », Journal of the Academy of Marketing Science, 23(4), pp. 335-345.

T. R. Wotruba (1991), «The Evolution of Personal Selling», Journal of Personal Selling and Sales Management, 11(2), pp. 1-12.

T. R. Wotruba (1995), «The Transformation of Industrial Selling: Causes and Consequences », Industrial Marketing Management, 25, pp. 327-338.

C. Yilmaz et S. D. Hunt (2001), «Salesperson Cooperation: The Influence of Relational, Task, Organizational, and Personal Factors », Journal of the Academy of Marketing Science, 29(4), pp. 335-357. 